\title{
Die Politisierung der Gemeinsamen Versammlung der Europäischen Gemeinschaft für Kohle und Stahl: Anfänge transnationaler Fraktionsbildung im Europäischen Parlament
}

\author{
Jürgen MITTAG
}

\section{Einleitung: Die Fraktionen des Europäischen Parlaments in der Forschung1}

Wenn sich im 21. Jahrhundert der Blick auf die Entwicklung des Europäischen Parlaments (EP) richtet, stehen in der Regel dessen Funktionen und Kompetenzgewinne im Mittelpunkt, weitaus seltener hingegen die politischen Binnenstrukturen. ${ }^{2}$ Rückt hingegen das Innenleben ins Blickfeld, wird den Fraktionen des Europäischen Parlaments, in denen sich die gewählten Europaabgeordneten nationaler Parteien zusammengeschlossen haben, eine wichtige Rolle zugeschrieben. Stärker als die so genannten „Parteien auf europäischer Ebene“ oder andere gesellschaftliche Akteure finden sie gleichermaßen in der politischen Berichterstattung wie auch in der wissenschaftlichen Forschung Berücksichtigung. ${ }^{3}$ Als zentrale Arbeitseinheiten des Europäischen Parlaments vereinen die Fraktionen nicht nur die gewählten Europaabgeordneten in Gruppierungen, die ähnliche politische Ziele verfolgen, sondern sie bündeln auch die Positionen der unterschiedlichen hier vertretenen nationalen Parteien.

Die Fraktionen des Europäischen Parlaments stellen einen Sonderfall transnationaler Repräsentation dar. Vergleicht man das EP mit anderen parlamentarischen Versammlungen jenseits des Nationalstaats, sticht mit Blick auf die Zahl der repräsentierten Nationalstaaten nicht nur der hohe Grad an transnationaler Institutionalisierung im Rahmen von Fraktionen, ${ }^{4}$ sondern aus historischer Perspektive auch deren vergleichsweise frühe Entstehung heraus. Während andere transnationale Versammlungen länderübergreifende Fraktionen entweder gar nicht kennen oder - wie im Fall

1. Für die Unterstützung der Archivrecherchen dieses Beitrags durch ein Reisestipendium ist der Autor der Fritz Thyssen Stiftung dankbar.

2. Vgl. allgemein zum Forschungsstand über das Europäische Parlament S. HIX, T. RAUNIO, R. SCULLY, Fifty Years On. Research on the European Parliament, in: Journal of Common Market Studies, 41(2003), S.191-202.

3. Vgl. als Forschungsüberblicke J. MITTAG, Chance oder Chimäre? Die grenzüberschreitende Interaktion politischer Parteien in Europa, in: DERS. (Hg.), Politische Parteien und europäische Integration. Entwicklung und Perspektiven transnationaler Parteienkooperation in Europa, Klartext Verlag, Essen, 2006, S.13-60; T. RAUNIO, Political Parties in the European Union, in: K. E. JØRGENSEN, M.A. POLLACK, B. ROSAMOND (Hg.), Handbook of European Union Politics, Sage, London, 2007, S.247-262.

4. Vgl. grundlegend S. MARSCHALL, Transnationale Repräsentation in Parlamentarischen Versammlungen. Demokratie und Parlamentarismus jenseits des Nationalstaates, Nomos, Baden-Baden, 2005. 
der Parlamentarischen Versammlung des Europarats, in der sich Fraktionen formal erst in den 1960er Jahren bildeten - einen weitaus schwächeren Grad an parteipolitischer Zusammenarbeit aufweisen, ${ }^{5}$ ist das Europäische Parlament von Anfang an durch ein hohes Maß an transnationaler Repräsentation gekennzeichnet. ${ }^{6}$

Die Fraktionen des Europäischen Parlaments sind annähernd so alt wie das Parlament selbst. Seit 1953 haben sich die zunächst nicht direkt gewählten, sondern aus den nationalen Parlamenten der Mitgliedstaaten in die EGKS-Versammlung delegierten Abgeordneten formal in politischen Gruppierungen zusammengeschlossen. Die lockere Zusammenarbeit der Parlamentarier im Rahmen von anfänglich lediglich drei Fraktionen verfestigte sich in den folgenden Jahren immer weiter, während zugleich die Fraktionsgröße im Zuge der Erweiterungsrunden beständig zunahm.

Vor dem Hintergrund dieser Ausgangsbeobachtungen stellt sich die Frage, welche allgemeinen Rahmenbedingungen und welche spezifischen Impulse dazu beigetragen haben, dass sich im Europäischen Parlament bzw. in der Gemeinsamen Versammlung der EGKS vergleichsweise früh Fraktionen gebildet haben und zum zentralen Strukturmerkmal der politischen Arbeit avanciert sind. Dieser Problemkomplex verdient vor allem deswegen Beachtung, weil im 1952 in Kraft getretenen EGKS-Vertrag keine Regelung über potenzielle Fraktionen der Gemeinsamen Versammlung vorgesehen war. Damit existierte auch keine Festlegung, ob sich die Abgeordneten entlang nationaler oder politischer Orientierungen gruppieren sollten. Im Gegensatz zur parlamentarischen Versammlung des Europarats und anderer transnationaler Zusammenschlüsse formierten sich dann aber bereits unmittelbar nach Zusammentreten der EGKS-Versammlung politische Gruppierungen, die im Zuge einer Neufassung der Geschäftsordnung im Juni 1953 dann auch rechtlich verankert wurden.

In der wissenschaftlichen Forschung hat die frühe Fraktionsbildung der Gemeinsamen Versammlung bislang wenig Beachtung gefunden. Während in älteren politikwissenschaftlichen Arbeiten der 1960er und 70er Jahre zum seinerzeit noch nicht direkt gewählten EP und seinen Fraktionen auch Untersuchungen zur Entsendung der

5. Siehe hierzu etwa die Ausführungen von Ernst B. Haas, der mit Blick auf den Europarat die ,nationale Partei“ als „Träger der Loyalität“ ausmachte. E.B. HAAS, Consensus Formation in the Council of Europe, Stevens, London, 1960, S.66.

6. Zur terminologischen Vereinfachung wird die erst mit der Einheitlichen Europäischen Akte rechtlich verankerte Bezeichnung „Europäisches Parlament“, die gleichwohl bereits in den Gesamtberichten der EWG-Kommission seit 1958 verwendet wurde, auch für die Phase der Gemeinsamen Versammlung der EGKS (1952-58) verwendet bzw. für den Zeitraum der Versammlung von EGKS, EWG und Euratom (offiziell 1958-87); die Versammlung selbst bezeichnete sich - je nach Sprachfassung - seit 1958/62 als Europäisches Parlament. 
Abgeordneten ${ }^{7}$ und zur Fraktionsbildung angestellt wurden, ${ }^{8}$ zeichnet sich seit etwa zwei Dekaden eine starke, fast einseitige Konzentration der Forschung auf das Abstimmungsverhalten der Europaabgeordneten sowie - damit verbunden - auf die Frage nach der inneren Geschlossenheit der Fraktionen ab. ${ }^{9}$ In diesen Studien zur Kohäsion der EP-Fraktionen ist der Zeitabschnitt vor der Direktwahl fast gänzlich aus dem Blickfeld verschwunden. ${ }^{10}$ Allein über die „Hintertür“ von Analysen zum Charakter der transnationalen Repräsentation auf europäischer Ebene, die in jüngster Zeit neues Interesse gefunden hat, wurde die Bedeutung der Gemeinsamen Versammlung zumindest punktuell wieder ins Licht der Forschung gerückt. ${ }^{11}$

Seitens der historischen Forschung sind diese Leerstellen bislang nicht einmal in Ansätzen gefüllt worden. Obgleich die historische Parlamentarismusforschung lange Traditionslinien aufweist, haben Fragen nach der Bedeutung transnationaler Verflechtungsprozesse bisher kaum Beachtung gefunden. Namentlich die parlamentarischen Versammlungen wurden als nachrangige Akteure der internationalen Beziehungen betrachtet. Systematische Studien zur transnationalen Repräsentation von Parlamenten entstanden erst in den 1990er Jahren. ${ }^{12}$ Diese Bemühungen sind in jüngster Zeit aufgegriffen und weiterentwickelt bzw. in einzelnen Fallanalysen vertieft worden..$^{13}$ All dies blieb aber weitgehend punktuell - und auch nur begrenzt auf die europäische Integration und ihre historische Dimension bezogen. Infolgedessen lässt sich resümierend festhalten, dass die „Parlamentarischen Versammlungen noch

7. Besondere Beachtung verdienen in diesem Zusammenhang H.-V. SCHIERWATER, Parlament und Hohe Behörde der Montanunion, Quelle und Meyer, Heidelberg, 1961 und P. REICHEL, Bundestagsabgeordnete in europäischen Parlamenten. Zur Soziologie des europäischen Parlamentariers, Westdeutscher Verlag, Opladen, 1974.

8. Vgl. etwa D. SPERLING, Der parlamentarische Charakter europäischer Versammlungen. Studie zur Entwicklung eines europäischen Parlamentarismus, Sythoff, Leiden, 1961; P.J.G. KAPTEYN, L'assemblée commune de la Communauté Européenne du Charbon et de l'Acier. Un essai de parlementarisme européen, Sythoff, Leiden, 1962; J. FITZMAURICE, The Party Groups in the European Parliament, Saxon House, Portsmouth, 1975.

9. Siehe zu diesem Forschungsstrang exemplarisch S. HIX, C. LORD, Political Parties in the European Union, Macmillan, Basingstoke (u.a.), 1997 und S. HIX, A.G. NOURY, G. ROLAND, Democratic Politics in the European Parliament, Cambridge University Press, Cambridge, 2007.

10. Vgl. als Ausnahmen T. OPPELAND, Das Parteiensystem der Europäischen Union, in: O. NIEDERMAYER, R. STÖSS, M. HAAS (Hg.), Die Parteiensysteme in Westeuropa, VS Verlag für Sozialwissenschaften, Wiesbaden, 2006, S.543-563; S. GUERRIERI, The start of European integration and the parliamentary dimension: the Common Assembly of the ECSC (1952-1958), in: Parliaments, Estates and Representation, 1(2008), S.183-193.

11. Vgl. insbesondere B. RITTBERGER, Building Europe's Parliament. Democratic representation beyond the nation state, Oxford University Press, Oxford, 2005 und J. POLLAK, Repräsentation ohne Demokratie. Kollidierende Systeme der Repräsentation in der Europäischen Union, Springer, Wien, 2007.

12. Vgl. E. KUPER, U. JUN (Hg.), Nationales Interesse und integrative Politik in transnationalen parlamentarischen Versammlungen, Westdeutscher Verlag, Opladen, 1997.

13. Vgl. etwa C. LOTTER, Die Parlamentarische Versammlung der Westeuropäischen Union. Demokratische Kontrolle europäischer Sicherheitspolitik, Nomos, Baden-Baden, 1997. 
nicht in ihrer Rolle als repräsentative Körperschaften wahrgenommen worden sind". 14

Vor diesem Forschungshintergrund stellt sich der vorliegende Beitrag der Aufgabe, auf dem Fundament der älteren Literatur und mittlerweile verfügbarer archivalischer Quellen die Frage nach den Ursachen für die frühe Fraktionsbildung im EP neu zu stellen und die ersten Entwicklungsetappen der Fraktionen zu beleuchten. Herangezogen werden dabei sowohl die stenographischen Protokolle der Gemeinsamen Versammlung als auch archivalische Quellen parteipolitischer und individueller Provenienz. ${ }^{15}$

\section{Historischer Hintergrund: Die Etablierung der EGKS-Versammlung}

Am 10. September 1952 trat das „Parlament“ der Montanunion - die Gemeinsame Versammlung - erstmals zusammen. Sie war erst im Verlauf der EGKS-Verhandlungen, vor allem auf deutsches Drängen hin, konzipiert worden, um der Hohen Behörde ein parlamentarisches Kontrollorgan gegenüber zu stellen. ${ }^{16}$ Es ist bereits mehrfach darauf verwiesen worden, dass in erster Linie föderalistische Vorstellungen seitens der deutschen Delegation Einfluss auf die Etablierung, wenngleich auch nicht auf die kompetenzielle Ausgestaltung der Gemeinsamen Versammlung, ausgeübt haben. So hatte etwa Walter Hallstein in einem Gespräch mit Jean Monnet in Paris am 27. Juli 1950 hinsichtlich der deutschen Vorstellungen erklärt:

„Wir haben [...] daran gedacht, den Ministerrat mit der Assemblée Commune zu einer Art Kongress im Sinne des amerikanischen Staatsrechts zusammenzufassen, bestehend aus einem Staatenhaus und einem nationalen Parlament, also hier einem supranationalen europäischen Parlament“.${ }^{17}$

Die Kompetenzen der Gemeinsamen Versammlung waren, nicht zuletzt infolge der deutlich zurückhaltenderen französischen und niederländischen Positionen, zunächst auf beratende und kontrollierende Funktionen begrenzt, während in der Rechtsetzung

14. S. MARSCHALL, op.cit., S.28.

15. Namentlich aus dem Archiv der sozialen Demokratie in Bonn (AdsD) und dem Archiv für ChristlichDemokratische Politik (ACDP) in Sankt Augustin.

16. Vgl. B. RITTBERGER, op.cit., S.79 und H.J. KÜSTERS, Die Verhandlungen über das institutionelle System zur Gründung der Europäischen Gemeinschaft für Kohle und Stahl, in: K. SCHWABE (Hg.), Die Anfänge des Schuman-Plans 1950/51/The Beginnings of the Schuman-Plan, Nomos, Baden-Baden (u.a), 1988, S.73-102.

17. Zit. nach D. KOSTHORST, M.F. FELDKAMP (Bearb.): Akten zur Auswärtigen Politik der Bundesrepublik Deutschland (AAPD) 1949/50, Oldenbourg Verlag, München, 1997. Dok. Nr. 99, Besprechung beim Vorsitzenden der Konferenz über den Schuman-Plan, Monnet in Paris, 27. Juli 1950, S.283, Fußnote 3. Der Verweis bei G. THIEMEYER, Die Debatten um die Versammlungen. Parlamentarismus und Demokratie in der Frühphase der europäischen Integration, in: J. MITTAG (Hg.), 30 Jahre Direktwahlen zum Europäischen Parlament, Nomos, Baden-Baden, 2011 (Im Erscheinen). 
keine (Mit-) Entscheidungsrechte gewährt wurden. Da sich die deutsche Delegation während der Verhandlungen über die Montanunion mit ihrem Vorschlag, die Versammlung direkt vom Volk wählen zu lassen, nicht hatte durchsetzen können, wurden die anfänglich 78 Abgeordneten - die sich nach Art. 20 des EGKS-Vertrags aus „Vertreter[n] der Völker der in der Gemeinschaft zusammengeschlossenen Staaten“ rekrutieren sollten - aus den Reihen der einzelnen nationalen Parlamente nach Straßburg delegiert. Mit dieser Praxis wurde das Prinzip des Doppelmandats etabliert, das dazu führte, dass die Europaabgeordneten bis 1979 ausnahmslos nationale Parlamentarier waren, die zusätzlich ein Mandat im Europäischen Parlament wahrnahmen - und infolgedessen auch erhebliche Unterschiede im Entsendungsmodus aufwiesen. Die gravierendsten Abweichungen betrafen dabei die Mandatsdauer, die Aufteilung der Mandate auf die Kammern der jeweiligen Legislative und den Bestellungsmodus: Im Gegensatz zum deutschen, niederländischen und belgischen System der Orientierung an der Fraktionsstärke wurden die Straßburger Parlamentarier in anderen Staaten von der jeweiligen Kammer mit Mehrheit gewählt, in Italien sogar mit absoluter Mehrheit. Das Veto der sozialdemokratischen und bürgerlichen Parteien verhinderte lange Zeit u.a. die Präsenz rechtsextremer oder kommunistischer Abgeordneter aus Frankreich oder Italien im Europäischen Parlament.

\section{Rechtliche Rahmenbedingungen: Debatten im Geschäftsordnungsausschuss}

Da im EGKS-Vertrag keine Aussagen über politische Gruppierungen getroffen wurden, blieb es einer Geschäftsordnung vorbehalten, hier nähere Regelungen zu treffen. Von zentraler Bedeutung für die Entwicklung der Fraktionen auf europäischer Ebene war infolgedessen die Geschäftsordnungsautonomie der Gemeinsamen Versammlung, die es ihr erlaubte, ohne Zustimmung des Rates über ihre inneren Angelegenheiten zu befinden - sofern dem keine spezifischen primärrechtlichen Regelungen entgegenstanden. ${ }^{18}$ Die Mitglieder der EGKS-Versammlung machten von der Möglichkeit des Selbstorganisationsrechts reichlich Gebrauch. Die ersten Sitzungen der Versammlung waren geprägt von ebenso regen wie kontroversen Debatten über Geschäftsordnungs- und Formaliafragen; der am 13. September 1952 eingesetzte Organisationsausschuss avancierte infolgedessen neben dem Geschäftsordnungsausschuss in den ersten Monaten zum wichtigsten Organ der Gemeinsamen Versammlung. ${ }^{19}$

18. Dazu zählte auch ein uneingeschränktes Budget-Recht über den eigenen Haushalt. Vgl. hierzu M. FORSYTH, Das Parlament der Europäischen Gemeinschaft. Die Bedeutung des Europäischen Parlaments für den demokratischen Charakter eines Vereinigten Europa, Europa Union Verlag, Köln, 1964.

19. Siehe hierzu auch den Victor Emanuel Preusker vorlegelegten „Bericht im Namen des Organisationsausschusses über die der Gemeinsamen Versammlung zu unterbreitenden Vorschläge über die Zahl, Zusammensetzung und Zuständigkeiten der für eine erfolgreiche Arbeit erforderlichen Ausschüsse“, in: Historical Archives of the European Union (HAEU), Common Assembly of the ECSC, AC.AP-RP/ORGA.1952 Rapports parlementaires de la commission d'organisation, AC 100. 
Während in der ersten Geschäftsordnung der Gemeinsamen Versammlung, die am 13. Januar 1953 in Kraft trat, zunächst nur Ausschüsse, aber keine Gruppen oder Fraktionen vorgesehen waren, ${ }^{20}$ wurde fünf Monate später ein entsprechender Passus ergänzt. Diesem Schritt waren mehrere Verhandlungsrunden in den Ausschüssen der EGKS-Versammlung vorangegangen. Zunächst hatte sich der Haushalts- und Verwaltungsausschuss in seiner Sitzung am 20. und 21. Februar 1953 mit der Thematik befasst und auf eine bereits bestehende Arbeitspraxis der politischen Kooperation verwiesen. In einem Schreiben an Jean Monnet vom 26. Februar 1953 resümierte H. Martin Blank als Ausschussvorsitzender, dass die

„Mehrzahl der Abgeordneten [...] offensichtlich der Auffassung [ist], dass die Bildung von Fraktionen auf supranationaler Ebene einen wesentlichen Beitrag zur Förderung der Arbeiten der Versammlung und damit zur Festigung der Europäischen Gemeinschaft darstellt. Tatsächlich haben sich inzwischen drei Fraktionen gebildet" ${ }^{21}$

Da der Haushalts- und Verwaltungsausschuss die Ansicht vertrat, ,dass es der Aufnahme einer entsprechenden Bestimmung in die Geschäftsordnung bedarf, um die Rechtsstellung der Fraktionen im Rahmen der Versammlung zu sichern",22 wurde die Angelegenheit an den Geschäftsordnungsausschuss überwiesen.

Dieser befasste sich erstmals in seiner Sitzung am 11. März 1953 mit der Thematik - und rekurrierte dabei auf nationalstaatliche Erfahrungen. Erstellt wurde ein Dossier zu den unterschiedlichen Modi der Fraktionsbildung in den zweiten Kammern der Parlamente in Deutschland, Frankreich und Italien sowie zum Senat der vierten französischen Republik (Conseil de la République). ${ }^{23}$ Dieses Dossier diente dem Geschäftsordnungsausschuss als Vorlage, um über die Aufnahme einer entsprechenden Ergänzung der Geschäftsordnung einen Konsens herzustellen.

Nach eingehenderen Debatten verständigte sich der Ausschuss auf die Formulierung: „Die Mitglieder der Versammlung können sich zu Gruppen nach ihren verschiedenen politischen Richtungen zusammenschließen". ${ }^{24}$ Dass diese Formulierung nicht ohne Kontroversen erzielt wurde, ist dem Ausschussprotokoll nur indirekt zu entnehmen; dass aber weder von Abgeordneten noch von Fraktionen die Rede ist, weist zumindest auf die Zurückhaltung hin, die bei der Formulierung an den Tag gelegt wurde. Wie strittig weitergehende Überlegungen waren, zeigt der Umstand, dass die vom deutschen sozialdemokratischen Abgeordneten Gerhard Kreyssig vorgeschlagene Ergänzung eines Passus zur Finanzierung von Fraktionen zurückgewiesen wurde.

20. Vgl. Europäische Gemeinschaft für Kohle und Stahl: Geschäftsordnung der Gemeinsamen Versammlung, o.O., März 1953, S.32 ff.

21. AdsD, Nachlass Gerhard Kreyssig, Abgeordnetentätigkeit EGKS, GV, Kopie des Schreibens von H. Martin Blank an Jean Monnet, 26.02.1953, S.2.

22. Ibid.

23. Eine Kopie dieses Dossiers vom 9. März 1953 im AdsD, Nachlass Gerhard Kreyssig, Abgeordnetentätigkeit EGKS, GV.

24. Ibid. 
Es sollte noch fast ein weiteres Vierteljahr dauern, bis eine endgültige Formulierung zur rechtlichen Stellung von Fraktionen gefunden wurde. In der Sitzung des Geschäftsordnungsausschusses am 8. Juni stand der Punkt „Organisation der Abgeordneten in Fraktionen " erneut auf der Tagesordnung. ${ }^{25}$ Ohne eingehendere Debatte $^{26}$ wurde von den fünf anwesenden Ausschussmitgliedern in dieser Sitzung die Formulierung angenommen, die dann auch vom Plenum der Gemeinsamen Versammlung verabschiedet wurde:

„1. Die Abgeordneten können aufgrund ihrer politischen Zugehörigkeit Fraktionen bilden. 2. Die Fraktionen sind gebildet, nachdem dem Präsidenten der Versammlung eine Erklärung über die Gründung zugeleitet worden ist, die die Bezeichnung der Fraktion, die Unterschrift ihrer Mitglieder sowie die Benennung ihres Büros enthalten muss. Diese Erklärung wird veröffentlicht. 3. Niemand darf auf der Mitgliederliste von mehreren Fraktionen stehen. 4. Die Mindestzahl der notwendigen Mitglieder für die Bildung einer Fraktion ist auf neun festgesetzt". .27

Die Modifikation der Geschäftsordnung vom Juni 1953 erhielt dann auch exakt diesen Passus in einem zusätzlich eingefügten Artikel 33a. ${ }^{28}$ In der Sitzung der EGKS-Versammlung vom 16. Juni 1953 wurde ohne Debatte den Ausführungen des Berichterstatters Paul Struye namens des Geschäftsordnungsausschusses zur Rolle von Fraktionen zugestimmt und der Antrag einstimmig angenommen. ${ }^{29}$ Mit dieser Regelung war der Weg für die Ausrichtung der Binnenstrukturen des Parlaments entlang politischer Orientierungen vorgezeichnet. Offiziell erhielten drei Fraktionen einen entsprechenden Status: die christlich-demokratische Fraktion, die sozialistische Fraktion sowie die Fraktion der Liberalen und Nahestehenden.

\section{Politische Impulse: Kontroversen um die Wahl des Präsidenten der Versammlung}

Schien die Bildung von Fraktionen mit Blick auf die Verhandlungen im Geschäftsordnungsausschuss primär eine technische Anpassung der Geschäftsordnung darzustellen, ergibt sich bei einer Betrachtung der Plenumssitzungen der EGKS-Versammlung ein anderes Bild. Bereits unmittelbar bei der Konstituierung der Gemeinsamen

25. AdsD, Nachlass Gerhard Kreyssig, Abgeordnetentätigkeit EGKS, GV, Protokoll der Ausschusssitzung, 08.06.1953. Aufgrund der geringen Zahl von Anwesenden wurde das formal notwendige Quorum zur Abstimmung zunächst nicht erreicht.

26. Seitens des Ausschusses wurde lediglich erörtert, ob - in Anlehnung an einige nationale Systeme jede Fraktion eine politische Erklärung abzugeben habe. Dieser Vorschlag fand jedoch keine Mehrheit.

27. Zit. nach ibid., S.2.

28. Vgl. Europäische Gemeinschaft für Kohle und Stahl: Geschäftsordnung der Gemeinsamen Versammlung, o.O., März 1953; die Modifikation wurde der gedr. Geschäftsordnung als „Berichtigung" beigefügt.

29. Verhandlungen der Gemeinsamen Versammlung. Ausführliche Sitzungsberichte, Sitzung vom 16. Juni 1953, S.48-49, in: Amtsblatt der Europäischen Gemeinschaft für Kohle und Stahl. 
Versammlung, als die Abgeordneten über die Wahl des Präsidenten abstimmten, zeichneten sich hier stärker politisch induzierte Kontroversen ab.

In der Eröffnungssitzung am 10. September 1952 und in der ersten Sitzung am 11. September hatte zunächst noch der italienische Senator und Christdemokrat Antonio Boggiano-Pico der Versammlung als Alterspräsident vorgesessen; in der zweiten Sitzung desselben Tages stand dann aber die Wahl eines Parlamentspräsidenten an. Diese Wahl hatte bereits im Vorfeld zu einigen Turbulenzen geführt, denn sowohl auf französischer als auch auf deutscher Seite hatten prominente Kandidaten ihr Interesse signalisiert. Unter den Franzosen hatte François de Menthon, der als Parteigänger Charles de Gaulles und Gründungsmitglied des Mouvement républicain populaire (MRP) eine wichtige Rolle in der französischen Innenpolitik spielte, der aber auch als Nachfolger Paul-Henri Spaaks zum Präsidenten der Versammlung des Europarats gewählt worden war, Interesse an diesem Amt bekundet. Er verzichtete dann jedoch auf eine Kandidatur, da er bereits in der Versammlung des Europarats das Präsidentenamt innehatte. ${ }^{30}$ Auf deutscher Seite war es hingegen der Vorsitzende der christlich-demokratisch/christlich-sozialen Unionsfraktion im Bundestag, Heinrich von Brentano, der Ambitionen angemeldet hatte. Von Brentano galt als „Chefdelegierter der CDU-CSU in Straßburg “31 und als erklärter Befürworter der europäischen Einigung; gegenüber der Perspektive einer hervorgehobenen Rolle der deutsch-französischen Beziehungen im Kontext der europäischen Integration legte er allerdings zunächst eine gewisse Reserve an den Tag. ${ }^{32}$ Da die christlich-demokratisch-konservativen Abgeordneten indes eine Mehrheit in der Versammlung stellten, schien die Wahl eines Abgeordneten dieser Strömung ausgemacht zu sein - mit Blick auf Jean Monnets Rolle als Präsident der Hohen Behörde und die Besetzung des Gerichtshofs mit einem Italiener war auch die Bestellung eines deutschen Politikers als Präsident der EGKS-Versammlung weitgehend unstrittig.

Als die Wahl anstand, trat aber der führende belgische Sozialist, Paul-Henri Spaak, gegen Heinrich von Brentano an. Die Kandidatur des Sozialisten Spaak wurde als Überraschung gewertet, hatte er selbst doch gegenüber der Zeitung Le Monde am Vorabend der Wahl noch eine Kandidatur verneint und betont, dass „er den Vorsitz nur übernehmen würde, wenn das helfen würde, ein Hindernis zu überwinden“. ${ }^{33}$ Der Ausgang der Wahl bot dann eine weitere Überraschung. Anders als von politischen Beobachtern erwartet, gewann Spaak und nicht Brentano die Wahl zum Präsidenten. Notwendig war im ersten Wahlgang eine absolute Mehrheit der abgegebenen Stim-

30. Von de Menthon selbst wurde aber der Antrag eingebracht, dass das zu wählende Präsidium der EGKS-Versammlung nur bis zu Beginn der nächsten ordentlichen Sitzungsperiode im Mai 1953 gewählt sei; dem Antrag wurde zugestimmt.

31. So Max von Brück in: Die Zeit, 09.04.1953.

32. Vgl. für eine europapolitische Einordnung Heinrich von Brentanos und zeitgenössische Urteile vor allem J. ELVERT, Heinrich von Brentano. Vordenker einer Konstitutionalisierung Europas, in: R. $\mathrm{KOCH}(\mathrm{Hg})$, Heinrich von Brentano. Ein Wegbereiter der europäischen Integration, Oldenbourg, München, 2004.

33. J. SCHWOEBEL, M. Paul-Henri Spaak est élu président de l'Assemblée de la communauté du charbon et de l'acier, in: Le Monde, 12.09.1952, S.1. Vgl. des Weiteren die Zeitung Europa-Union, September 1952, S.3. 
men. Von den abgegebenen 69 Stimmen war eine Stimme ungültig, so dass 35 Stimmen die absolute Mehrheit bildeten. Insgesamt 30 Stimmen wurden für von Brentano abgegeben, 38 Stimmen entfielen auf Spaak, der damit gewählt war. ${ }^{34}$

Das Resultat lässt sich darauf zurückführen, dass es Spaak gelungen war, die Stimmen aller französischen, belgischen und luxemburgischen Parlamentarier der Versammlung auf sich zu vereinigen und zusätzlich noch die Stimmen der niederländischen, italienischen und deutschen Sozialisten bzw. Sozialdemokraten sowie von zwei liberalen Abgeordneten aus Italien zu mobilisieren. ${ }^{35} \mathrm{Im}$ Wahlergebnis spiegelten sich damit die divergierenden Loyalitäten der Abgeordneten zwischen einerseits nationaler und andererseits politischer Orientierung wider. Hatten die primär frankophonen Abgeordneten aus Frankreich, Belgien und Luxemburg nach Ländererwägungen abgestimmt, votierten Niederländer, Deutsche und Italiener aufgrund politischer Erwägungen. ${ }^{36}$ Zweifellos hatte Spaaks beträchtliches europapolitisches Ansehen eine wichtige Rolle gespielt. Der ehemalige belgische Premier genoss als früherer Präsident der ersten Vollversammlung der UN, als spiritus rector des Benelux-Verbunds und als erster Präsident der Beratenden Versammlung des Europarats ein hohes internationales Ansehen, das vor allem durch seine Rücktrittsrede als Präsident der Europaratsversammlung noch verstärkt worden war, deren begrenzte Kompetenzen er mit den Worten ,heute ist es nicht mehr diese Versammlung, welche die Sache des Vereinigten Europa vertritt", harsch kritisiert hatte. ${ }^{37}$

Das Ergebnis der Präsidentenwahl führte unter den deutschen Delegierten der Versammlung zu einem Eklat. Franz-Josef Strauß, als junger Bundestagsabgeordneter Mitglied der EGKS-Versammlung, wurde vom CDU-Organ „Union in Deutschland" mit den Worten zitiert:

„Die SPD soll es nie mehr wagen, sich zum Verfechter der Gleichberechtigung [Deutschlands] aufzuwerfen, denn sie hat in einer entscheidenden Situation die deutsche Solidarität durchbrochen". 38

Seitens der im Bundestag oppositionellen SPD griff man diesen Vorwurf auf, um Strauß vorzuhalten,

„dass diese Beschwerde seltsam im Munde eines Mannes wirke, dessen Partei der SPD immer wieder Mangel an europäischer Gesinnung und überspitzten Nationalismus vorgeworfen habe. Wenn man den europäischen Gedanken ernst nehme und damit auch den eines europäischen Parlaments, dann werde man sich auch folgerichtig mit dem Entstehen

34. Die übrigen Mitglieder der Versammlung, überwiegend italienische Abgeordnete, waren nicht anwesend.

35. Vgl. für diese Klassifizierung des Abstimmungsergebnisses: AdsD, Sammlung Personalia, Spaak, Paul-Henri, FP, 12.09.1952.

36. Deutschland, Frankreich und Italien waren mit je 18 Delegierten, Belgien und die Niederlande mit 10, Luxemburg mit 4 Vertretern in der Gemeinsamen Versammlung vertreten.

37. Vgl. zu dieser Wertung auch den Kommentar in: Stuttgarter Nachrichten, 12.09.1952.

38. Union in Deutschland, 17. September 1952. 
europäischer Fraktionen befreunden müssen, die [...] international zusammengesetzt sein können, wenn sie es auch nicht zu sein brauchen“. 39

Jenseits der innenpolitischen Debatten in Westdeutschland hatte die Kontroverse um die Präsidentenwahl aber auch Auswirkungen auf die Fraktionsbildung in der Gemeinsamen Versammlung. Nicht durch schriftliche Quellen belegt, aber in mehreren Interviews zum Ausdruck gebracht wurde die Sicht, dass sich bereits am 11. September 1952, also im Zuge der ersten Abstimmungen der EGKS-Versammlung, informell Fraktionen bildeten. So verweist u.a. Heribert Krämer auf ein entsprechendes Statement des Generalsekretärs der christlich-demokratischen Fraktion. ${ }^{40}$ Und auch Franz Heidelberg betont in seiner Pionierstudie zum Europäischen Parlament, dass dieser Vorgang ,die Entwicklung von Fraktionen beschleunigt hat“ ${ }^{41}$ In den folgenden Monaten wurde auch im Plenum - so etwa in der außerordentlichen Sitzung der Versammlung im März 1953 - wiederholt von politischen Gruppen und deren Vorsitzenden gesprochen. Und in der Sitzung der EGKS-Versammlung am 16. Juni 1953, in dem der Ausschussvorschlag zur Bildung von Fraktionen formal überhaupt erst angenommen worden war, stellte der Präsident fest:

„Ich bitte die Vorsitzenden der bereits bestehenden Fraktionen, dem Präsidium so bald wie möglich die Gründungserklärung zu übergeben, deren Erfordernisse von der Versammlung soeben beschlossen wurden" . ${ }^{42}$

Dass neben der nationalen Herkunft auch die parteipolitische Orientierung von Anfang an eine gewisse Bedeutung in der EGKS-Versammlung spielte, hatte sich auch bei der Wahl der Vizepräsidenten gezeigt, die in der nachfolgenden Sitzung am selben Tage vorgenommen wurde. Nachdem mit Spaak ein Belgier zum Präsidenten gewählt worden war, votierte die Versammlung dergestalt für die Vizepräsidenten, dass jeder EGKS-Mitgliedstaat - außer Belgien - einen Vizepräsidenten stellte. Parteipolitisch stammten drei Vizepräsidenten aus dem christlich-demokratisch-konservativen Lager (Hermann Pünder (DE), Pierre-Henri Teitgen (FR), Gerrit Vixseboxse (NL)), einer aus der liberalen Parteienfamilie (Alesandro Casati (IT)) und einer besaß einen sozialistischen Hintergrund (Jean Fohrmann (LU)). Die politischen Mehrheitsverhältnisse der EGKS-Versammlung spiegelten sich damit auch in der Zusammensetzung des Präsidiums wider. Enthielt die erste Fassung der Geschäftsordnung zunächst noch keinen Hinweis auf die politische Orientierung der Vizepräsidenten, so wurde später in Artikel 7 der Geschäftsordnung festgelegt, dass im Fall einer Neu- oder Wiederbesetzung des Amts eines Vizepräsidenten die „Fraktion, der das Mitglied angehörte, dessen Sitz frei geworden ist, [...] einen Kandidaten als Ad-Interim Mit-

39. Zit. nach einer „Deutsche Solidarität“ und europäische Gesinnung“ übertitelten, maschinenschriftlichen Abschrift im AdsD, Sammlung Personalia, Paul-Henri Spaak.

40. H. KRÄMER, Die Stellung der politischen Parteien in der Völkerkammer eines künftigen Europaparlaments, Diss. Univ. Mainz, Mainz, 1956, S.126.

41. F.C. HEIDELBERG, Das Europäische Parlament. Entstehung, Aufbau, Erfahrungen und Erwartungen, August Lutzeyer, Baden-Baden/Bonn, 1959, S.32.

42. Verhandlungen der Gemeinsamen Versammlung. Ausführliche Sitzungsberichte, Sitzung vom 16. Juni 1953, S.49, in: Amtsblatt der Europäischen Gemeinschaft für Kohle und Stahl. 
glied des Präsidiums“ benennt. ${ }^{43}$ Die Rolle der Fraktionen wurde darüber hinaus dadurch aufgewertet, dass die Fraktionsvorsitzenden Mitglieder des Präsidialausschusses wurden und auf diesem Wege Einfluss auf die Tagesordnung der Versammlung bzw. des Parlaments nehmen konnten.

\section{Finanzielle Anreize: Die budgetäre Ausstattung der Fraktionen}

Bereits in den ersten Ausschussberatungen über die Rolle potenzieller Fraktionen hatten finanzielle Erwägungen eine wichtige Rolle gespielt. Im Zuge der Debatten über den Haushaltsplan der Gemeinsamen Versammlung für den Zeitraum 1953/54 kam diese Thematik erneut auf die Agenda. Seitens des Haushaltsausschusses hatte Pierre Vermeylen in der außerordentlichen Tagung am 11. März 1953 den Vorschlag unterbreitet, ,in den Haushaltsplan einen Ausgabenposten einzusetzen, der für die parlamentarische Arbeit benötigt wird“. ${ }^{44}$ Dieser Vorschlag basierte auf informellen Gesprächen, die seitens der christlich-demokratischen und sozialistischen Abgeordneten im Vorfeld über die Ausstattung geführt worden waren, während die liberalen Abgeordneten in diesem Punkt Zurückhaltung gezeigt hatten und aus diesem Grund von den Sprechern - dem Franzosen Guy Mollet für die Sozialisten und Sozialdemokraten sowie dem Niederländer Maan Sassen für die Christdemokraten - nicht zu weiteren Gesprächen hinzugezogen wurden. Nach eingehenderen Debatten und nachdem die liberale Gruppe festgehalten hatte, dass es sich nur ,um eine Übergangsregelung handelt", wurde Konsens über einen Kredit von 5 Millionen belgischen Francs an potenzielle politische Gruppen hergestellt. ${ }^{45}$

Dieser Betrag wurde indes kurz darauf um die Hälfte gekürzt, da mehrere Presseagenturen über eine zusätzliche Besoldung der Abgeordneten der EGKS-Versammlung kritisch berichtet hatten. ${ }^{46}$ In der Plenumssitzung vom 16. Juni 1953 wurde der Vorschlag dahingehend präzisiert,

,jeder der anerkannten, mindestens aus neun Mitgliedern bestehenden Gruppen einen festen Beitrag von 500.000 b. Fr. zuzüglich einer nach der zahlenmäßigen Stärke der Gruppe

43. Siehe M. FORSYTH, op.cit., S.50 f. Diese Änderung ist mutmaßlich auf den Tod des Präsidenten De Gasperi im August 1954 zurückzuführen. Sie wurde ohne Änderungsanträge von der Versammlung gebilligt. Vgl. hierzu die offizielle EP-Studie von F. PIODI, Die Ausschüsse der Gemeinsamen Versammlung. Studie über die wichtigsten Arbeiten der Ausschüsse der gemeinsamen Versammlung der EGKS im Zeitraum 1952-1958, OPOCE, Luxemburg, 1958, S.118.

44. Zit. nach H. KRÄMER, op.cit., S.128.

45. Vgl. die Debatte in der Plenumssitzung der Versammlung vom 11. März 1953. Verhandlungen der Gemeinsamen Versammlung. Ausführliche Sitzungsberichte, Außerordentliche Märztagung, Juni 1953, S.9-15, in: Amtsblatt der Europäischen Gemeinschaft für Kohle und Stahl; hier v.a. S.15.

46. Siehe hierzu die Ausführungen von Paul Struye in der Plenumssitzung der Versammlung vom 16. Juni 1953. Verhandlungen der Gemeinsamen Versammlung. Ausführliche Sitzungsberichte, Sitzung vom 16. Juni 1953, S.54 und 55, in: Amtsblatt der Europäischen Gemeinschaft für Kohle und Stahl. 
schwankenden und nach dem Verhältnis der eingetragenen Mitglieder errechneten Summe von 10.000 b.Fr. je Mitglied zu bewilligen“.

Dieser Vorschlag provozierte eine längere Kontroverse, in der nicht zuletzt das divergierende Selbstverständnis der Abgeordneten hinsichtlich ihrer eigenen Rolle, des Charakters der EGKS-Versammlung und deren politischer, nationaler und sonstiger Spannungslinien zum Ausdruck kam.

Der belgische Senator Paul Struye war in erster Linie auf Sparsamkeit bedacht und um die Außenwirkung besorgt, da er befürchtete, die Mittel könnten zweckentfremdet angelegt werden. Vor diesem Hintergrund sah er

„die Aufwendungen [...] angesichts der Hilfe, die [...] von der amtlichen Dienststelle, vom zuständigen Sekretariat zuteil wurde, in keinerlei Verhältnis stehen zu den Krediten, deren Annahme heute von Ihnen erbeten wird. Sie dürfen mir [Struye] glauben, dass ein solcher Beschluss sehr viel Ärger schaffen würde“"

Eine noch kritischere Sichtweise vertrat der Niederländer Albertus Hendrikus Korthals, der die Frage aufwarf, ob überhaupt politische Gruppen oder Fraktionen in der EGKS-Versammlung bestehen würden, da innerhalb der einzelnen Parteienfamilien doch „große Meinungsverschiedenheiten zutage treten“ würden. Seines Erachtens hätte man mit dem „Entwerfen bestimmter grundsätzlicher Programme und Pläne für die praktische Politik beginnen müssen, auf deren Grund sich dann die Gruppen hätten bilden können“.

Wie breit die Argumente der Gegner einer Fraktionsfinanzierung durch die EGKS gefächert waren, zeigt die Stellungnahme des aus den Niederlanden delegierten Abgeordneten Willem Rip. Er betrachtete die Fraktionsbildung und ihre Finanzierung als Angelegenheit der einzelnen nationalen Delegationen und forderte, dass ,die nationalen Gruppierungen aus den sechs Ländern für die Fraktionsbildung auf dieser Ebene Mittel zur Verfügung stellen“. Der Franzose Georges Laffargue argumentierte ähnlich, indem er erklärte:

„Wenn Sie glauben, dass die politischen Gruppen noch eine große Geschlossenheit zeigen, wenn Sie ihre Landesgrenzen verlassen haben, so geben Sie sich großen Täuschungen hin".

Die Befürworter einer Finanzierung von Fraktionen rekurrierten zum Teil auf die gleichen oder auf ähnliche Sachverhalte wie ihre Kontrahenten, wendeten diese aber argumentativ ins Gegenteil. So betonte der niederländische Abgeordnete Maan Sassen mit Blick auf demokratietheoretische Erwägungen:

„Wir haben zur Zeit keine europäischen politischen Parteien und werden sie auch in absehbarer Zeit nicht haben. Ich glaube daher, dass Kredite für unsere Gruppen durchaus gerechtfertigt sind“.

Im weiteren Verlauf der Debatte ergänzte Sassen seine Argumentation noch um den Hinweis auf die Notwendigkeit zur „Fühlungnahme“ auch in der Zeit zwischen den Sitzungsperioden und begründete diese mit den zahlreichen sachbezogenen Herausforderungen, mit denen die Abgeordneten der Versammlung konfrontiert seien. Von 
Herbert Wehner wurde auf die Entwicklungsperspektiven und -notwendigkeiten der EGKS-Versammlung verwiesen. Namentlich mit Blick auf die Kontrollfunktion der Versammlung sei, so Wehner, „durch Einrichtungen der Versammlung am Sitz der Hohen Behörde so viel wie möglich über die laufende Tätigkeit dieser Behörde und anderer Organe" an Informationen zu ermitteln. Im Gegensatz zu Sassen und anderen Befürwortern wies Wehner aber den Gedanken zurück, dass die Einrichtung von Fraktionen vor allem im Hinblick auf die Debatten um die Europäische Politische Gemeinschaft sowie die Einführung zukünftiger Direktwahlen zum Europäischen Parlament zu sehen sei. „Ich bin es selber gewesen“, so Wehner,

,der in dem damaligen provisorischen Organisationskomitee, der Versammlung, das in Luxemburg getagt hat, als sich unsere anderen Ausschüsse noch nicht konstituiert hatten, den Vorschlag zur Organisierung solcher Büros oder Verbindungsstellen gemacht hat. [...] Mein Wunsch ist, dass die Versammlung so wie sie jetzt ist, dasjenige, was ihr der Vertrag gestattet, bis an die äußerste Grenze ausschöpft. Dazu gehört eben, dass die Gruppen lebendig werden und nicht nur die Chance haben, sich zwischen diesen Tagungen kurz einmal über fundamentale Fragen zu unterhalten" . ${ }^{47}$

Am Ende dieses Schlagabtauschs, der eine der bis dahin grundlegendsten Debatten über die Rolle und Ausgestaltung der EGKS-Versammlung darstellt und damit die parlamentarische Dimension der europäischen Integration markiert, stand eine Annahme des Vorschlags, den Fraktionen den bereits als Kompromiss ausgehandelten reduzierten Betrag aus Haushaltsmitteln der Versammlung zu gewähren. Der Versuch des gaullistischen EP-Abgeordneten und französischen Senatsmitgliedes Michel Debré bei den nächsten Haushaltsberatungen im Januar 1954, den Etatansatz um weitere 50 Prozent zu reduzieren, fand keine Mehrheit. Zwei Jahre später, im März 1956, hatte man sich bereits arrangiert. Der Haushaltsansatz - und damit auch die Zuwendung an die Fraktionen - wurde ohne Aussprache angenommen.

\section{Parlamentarische Praxis: Sitzordnung und Infrastruktur}

Die Fraktionsbildung, die durch die Deklaration der Fraktionen zwischen dem 20. und 23. Juni 1953 auch offiziell vollzogen wurde, konzentrierte sich in den 1950er Jahren zunächst auf drei Fraktionen: eine christlich-demokratische Fraktion, eine sozialistisch-sozialdemokratische Fraktion sowie eine Fraktion der Liberalen und Nahestehenden (,apparentés“). Die Fraktionsbildung orientierte sich damit stark an den klassischen Spannungslinien (cleavages) und entsprach grob den ideologischen Mustern in den nationalen Parlamenten der EGKS-Mitgliedstaaten. Dass keine weiteren Fraktionen entstanden, ist in erster Linie auf den skizzierten Bestellungsmodus der Abgeordneten zurückzuführen, der das politische Spektrum auf die politische Mitte begrenzte. Zur Mitte des Jahrzehnts gab es nur noch zwei Parlamentarier der Versammlung, die sich keiner Fraktion angeschlossen hatten. Der eine, der niederländi-

47. Ibid., verschiedene Seiten. 
sche Liberale Albertus Hendrikus Korthals, erklärte, dass die mangelnde liberale Ausrichtung der Fraktion hierfür maßgeblich sei. ${ }^{48}$ Der andere, Michel Debré, versuchte sich hingegen in den Debatten durch eine gezielt unabhängige Positionierung zu profilieren. ${ }^{49}$

Die im Zuge der rechtlichen und finanziellen Regelungen zur Fraktionsbildung gefassten Entschlüsse fanden in der Folge ihr Korrelat in den Arbeitsprozessen der Gemeinsamen Versammlung. In den 1950er Jahren bildete sich dabei schrittweise eine Art transnationaler parlamentarischer Kultur heraus, die sich vor allem auf eine wachsende Kommunikation von Vertretern unterschiedlicher Nationalität stützte. So konnte Hermann Pünder als erster Vizepräsident der EGKS-Versammlung Konrad Adenauer mitteilen, dass im Straßburger Europahaus jeder

„Delegierte die schönste Gelegenheit [hat,] wertvolle Informationen zu sammeln. Sie sind umso wertvoller, als die Gesprächspartner - als Parlamentarier im Augenblick unmittelbarer Verantwortung enthoben - meist sehr viel freier und offener einem angesehenen Kollegen der anderen Nationen ihre Meinung äußern, als wenn sie sich in hohen Amtsstellungen befänden“. .50

Neben der Hilfestellung durch die Verwaltungen der nationalen Repräsentativversammlungen ${ }^{51}$ wurde die parlamentarische Kultur vor allem von den Fraktionen geprägt. Als wichtiges Merkmal ist dabei die Ausgestaltung der parlamentarischen Infrastruktur zu berücksichtigen, die eine zunehmende Professionalisierung der Fraktionsarbeit nach sich zog und - neben den gemeinsamen Fraktionssitzungen - sich vor allem in der Einrichtung eigenständiger Sekretariate, der Bildung von Arbeitsgruppen und der Nutzung eigener Räumlichkeiten widerspiegelte. Gerade die Fraktionen boten angesichts der zeitlich begrenzten Sitzungsperioden Gewähr für eine kontinuierliche Sacharbeit, da ,sie das ganze Jahre über regelmäßig zusammen[traten]" und, so der belgische EGKS-Parlamentarier Pierre Wigny in einer ersten systematischen Rückschau, sie sogar

„Arbeitsgruppen nach Sachgebieten gebildet [haben], in denen die Probleme der Gemeinschaft unter dem besonderen Gesichtswinkel dieser politischen Organisationen analysiert und erörtert werden". .52

Dass die Politisierung der EGKS-Versammlung und die Herausbildung einer transnationalen parlamentarischen Kultur jedoch auch an Grenzen stießen, dokumentieren die Überlegungen zur Sitzordnung. Ebenso wie in der Beratenden Versammlung des

48. Vgl. T. WESTERTERP, Europese Fractievorming, in: Internationale Spectator, Juli(1958), S.367.

49. Vgl. G.v. OUDENHOVE, The Political Parties in the European Parliament. The First Ten Years, Sijthoff, Leiden, 1965, S.28.

50. Bundesarchiv Koblenz (BAK), Nachlass Hermann Pünder, 005/00412, Hermann Pünder an Konrad Adenauer, 14.01.1953.

51. So hatte bereits Jean Monnet als Präsident der Hohen Behörde im Vorfeld der ersten Sitzung der Gemeinsamen Versammlung die Generalssekretäre der nationalen Parlamente zur Organisation der Präsidentenwahlen der EGKS-Versammlung herangezogen. Vgl. BAK, Nachlass Heinrich von Brentano, 1239/167, Jean Monnet an Heinrich von Brentano, 05.09.1952.

52. P. WIGNY, Die parlamentarische Versammlung im Europa der Sechs, o.O., o.J., [Luxemburg 1957], S.22. 
Europarats hatte man sich auch in der EGKS-Versammlung bei der Konstituierung für eine alphabetische Sitzordnung entschieden. Mit diesem Prinzip beabsichtigte man, einer zu starken Orientierung an Nationalitäten entgegenzuwirken. Nach der formalen Gründung von Fraktionen 1953 wurde die alphabetische Sitzordnung zunächst jedoch weiter beibehalten; erst im Zuge der Neu-Konstituierung der Versammlung - nach Inkrafttreten der Römischen Verträge 1958 - nahmen die Abgeordneten ihre Plätze in Anlehnung an ihre Fraktionsbindung ein. ${ }^{53}$ Über die Gründe sind vielfältige Spekulationen angestellt worden, lag doch eine Umgruppierung entlang der Fraktionszugehörigkeiten nahe. Als Erklärungsansatz für das Zögern wird u.a. das Argument herangezogen, dass eine eindeutige rechts-links-Ordnung vermieden werden sollte und sich zudem zunächst keine Fraktion bereit erklärte, den Platz auf der rechten Seite einzunehmen.

Die fehlende visuelle Abgrenzung dokumentierte gewissermaßen die zu Beginn der Fraktionsbildungen noch fragile Fraktionszugehörigkeit und ihre innere Heterogenität. So fand sich in der christlich-demokratischen Fraktion eine protestantische Minderheit, die neben den nationalen Delegationen ebenfalls eigene Treffen abhielt. Noch heterogener waren die Strukturen der Fraktion der Liberalen und Nahestehenden, über die Guy van Oudehove im Jahre 1965 konstatierte: „The criterion for membership of this group is seen to be a purely negative one, namely that of being neither a Socialist nor a Christian Democrat" ${ }^{54}$ Die liberale Fraktion des Europäischen Parlaments, die im Juni 1953 elf Mitglieder zählte, galt in den ersten Jahrzehnten als Auffangbecken von Abgeordneten unterschiedlichster Couleur rechts von der politischen Mitte. Die politische Bandbreite dieser Fraktion hatte zur Konsequenz, dass originär liberale Parteien sich lange Zeit in der Minderheit befanden, während anfangs die Gaullisten das Bild der Fraktion prägten. Vor diesem Hintergrund verzichtete die liberale Fraktion zunächst sowohl auf Arbeitsgruppen als auch auf ein eigenes $\mathrm{Pu}-$ blikationsorgan. ${ }^{55}$

Die sozialistische Fraktion mit 23 Mitgliedern im Juni 1953 erwies sich - trotz divergierender Positionen der Abgeordneten der beiden größten Parteien (der deutschen SPD und der französischen Sozialisten) - sowohl mit Blick auf ihre Zusammensetzung als auch hinsichtlich der europapolitischen Positionen als vergleichsweise stabil und kohärent. Bei der ersten offenen namentlichen Abstimmung im Mai 1955 votierte die sozialistische Fraktion ebenso wie die liberale Fraktion - aber im Gegensatz zu den Christdemokraten - einheitlich. ${ }^{56}$ In den 1950er Jahren wird der Fraktion zudem - ungeachtet der latenten Europaskepsis der SPD sowie der Kritik der französischen Sozialisten am christlich-demokratischen und kapitalistischen Europa der Sechs in der ersten Hälfte des Jahrzehnts - der mit Abstand dynamischste Part unter den drei Parlamentsfraktionen zugeschrieben. ${ }^{57}$ Zurückgeführt wird dies nicht zuletzt auf die Oppositionsrolle, die zahlreiche sozialistische und sozialdemo-

53. Vgl. hierzu G.v. OUDENHOVE, op.cit., S.138 ff.

54. Ibid., S.28.

55. Siehe M. FORSYTH, op.cit., S.64-65.

56. Vgl. G.v. OUDENHOVE, op.cit., S.50 f.

57. Vgl. M. FORSYTH, op.cit., S.56. 
kratische Parteien in ihren nationalen Parlamenten innehatten. Die gemeinsame europäische Fraktion wurde so zur ,wichtige[n] Plattform“, um Einfluss auf europäischer Ebene geltend zu machen. ${ }^{58}$ Besondere Bedeutung kam hierbei dem bereits 1953 in Luxemburg eingerichteten Sekretariat zu, das der Fraktion Serviceleistungen bot, jedoch nicht über das Recht verfügte, eigenständige Entscheidungen zu treffen. Vor allem nach der Gründung des „Verbindungsbüros der sozialistischen Parteien der Mitgliedstaaten der Europäischen Gemeinschaften und der sozialistischen Fraktion des Europäischen Parlaments" entwickelten sich hier rege transnationale Aktivitäten, da zahlreiche Sitzungen durchgeführt und gemeinsame Kongresse mit den sozialistischen und sozialdemokratischen Parteien der Mitgliedstaaten abgehalten wurden, die schließlich sogar zu einem gemeinsamen Programm führten. ${ }^{59}$

Die christlich-demokratische Fraktion (CD) stellte in den Gründungsjahrzehnten der Europäischen Gemeinschaft stets die stärkste Fraktion des Europäischen Parlaments - im Juni 1953 zählte sie 38 Mitglieder. Dies ist nicht zuletzt auf den Umstand zurückzuführen, dass in allen sechs Mitgliedstaaten der Gemeinschaft christlich-demokratische Parteien im nationalen Parteiensystem eine wichtige Rolle spielten. Lediglich die Bedeutung der französischen Vertretung in der christlich-demokratischen EP-Fraktion nahm in den 1960er Jahren aufgrund des Einflussverlustes des Mouvement républicain populaire deutlich ab. In den 1950er und 60er Jahren setzte sich die CD-Fraktion, in der nicht nur katholische Abgeordnete, sondern auch niederländische und deutsche Protestanten vertreten waren, in erster Linie für einen (föderalen) Ausbau der europäischen Integration ein, präsentierte sich aber in den Parlamentsdebatten und bei Abstimmungen - nicht zuletzt bei Agrarthemen - weniger geschlossen als die sozialistische Fraktion. Dennoch ging man auch in der christlichdemokratischen Fraktion rasch dazu über, die transnationale Fraktionsarbeit auszubauen. Schon im Juni 1953 wurden Ergebnisprotokolle von den Fraktionssitzungen angefertigt und ein regelmäßiger Zyklus von Sitzungen in Brüssel und Straßburg etabliert. ${ }^{60}$ Im November 1954 begann man, sich in der Fraktion dann sehr grundsätzlich mit der politischen Programmatik auseinanderzusetzen. So konstatierte Robert Houben seitens der belgischen Christdemokraten

„le groupe désire en effet préciser sa doctrine, afin d'accomplir au mieux sa mission en situant dans un ensemble et en considérant dans leurs répercussions à plus longue échéance les questions à l'égard desquelles il aura à prendre position". ${ }^{61}$

58. T. STAMMEN (Hg.), Parteien in Europa. Nationale Parteiensysteme, Transnationale Parteienbeziehungen, Konturen eines europäischen Parteiensystems, Bayerische Landeszentrale für politische Bildungsarbeit, München, 1978, S.264.

59. Vgl. hierzu grundlegend die Sozialistische Europa-Korrespondenz, v.a. vom 03.09.1961, Mikrofilm im AdsD und die ergänzenden Dokumente der HAEU, Bestand GSPE - Groupe socialiste du Parlement européen.

60. ACDP, 09-001-009/1, Procès-verbal de la réunion du groupe démocrate-chrétien, 16.06. 1953.

61. ACDP, 09-001-009/1, Houben an Opitz, 12.11.1954; Note au groupe démocrate-chrétien de l'assemblée commune. 


\section{Fazit: Triebkräfte und Hemmfaktoren der Politisierung der EGKS- Versammlung}

Verglichen mit den nationalstaatlichen Parlamentsfraktionen operierten die Fraktionen des Europäischen Parlaments von Anfang an in einer gänzlich differierenden institutionellen Umgebung, da es keine geschlossenen, hierarchisch organisierten Parteien gab, die das Fundament der Parlamentsfraktion bildeten. Vielmehr sind die Abgeordneten bis heute an die nationalen Parteien gebunden, von denen sie aufgestellt oder delegiert werden. Die soziale und kulturelle Heterogenität der nationalen Parteien spiegelt sich zwangsläufig auch in der Versammlung bzw. im Europäischen Parlament und in der inneren Vielfalt der Fraktionen wider. Dennoch ist es den Fraktionen schrittweise gelungen, ihre Rolle auszubauen, indem sie prozedurale Reformen einführten, die es ihnen erlaubten, sich der politischen Möglichkeiten und Ressourcen der Versammlung bzw. des Europäischen Parlaments zu bedienen. Damit haben die Fraktionen - trotz aller immer wieder konstatierten Fluktuation - sowohl an Profil als auch an Stabilität gewonnen.

Abschließend lässt sich ein ganzes Bündel an Ursachen für die vergleichsweise frühzeitige Politisierung der EGKS-Versammlung durch transnationale Fraktionsbildung identifizieren. Von grundlegender Bedeutung für die Dynamik der innerorganisatorischen Ausgestaltung war zweifellos die Autonomie in Geschäftsordnungsfragen. Der Umstand, dass ein ,uneingeschränktes und selbstständiges BudgetRecht" für den eigenen Etat bestand, ${ }^{62}$ erlaubte es der Versammlung, binnen kurzer Zeit eine sehr viel weiter entwickelte parlamentarisch-politische Infrastruktur zu etablieren, als dies in anderen transnationalen parlamentarischen Versammlungen der Fall war. Verstärkt wurde diese parlamentarische Ausgestaltung durch die politischen Konflikte im Zuge der Wahl des Präsidenten, aber auch durch die politisch-konzeptionellen Auseinandersetzungen im Zuge der Haushaltsdebatte der Versammlung. Die Notwendigkeit der Abgeordneten, sich mit dem eigenen Budgetentwurf auseinanderzusetzen, führte fast unweigerlich zu einer Reflexion über das Selbstverständnis und die künftige Rolle der Gemeinsamen Versammlung in der institutionellen Architektur der EGKS. Ungeachtet aller Differenzen zeichnete sich hier ein partei- und länderübergreifender Konsens ab, die EGKS-Versammlung im Sinne traditioneller nationaler Parlamentarismusleitbilder weiterzuentwickeln und ihr zu diesem Zweck auch die notwendigen Mittel an die Hand zu geben - zu denen auch das Instrumentarium der Fraktionen gezählt wurde.

Die einmal etablierten Fraktionen entwickelten in der Folge beträchtliche Aktivitäten. In dem Augenblick, in dem Personal, Räume und Ressourcen zur Verfügung standen, wurde diese parlamentarische Infrastruktur von den Abgeordneten auch genutzt. Im Sinne eines sich selbst tragenden Prozesses führte diese Ausstattung zu einer weiteren Politisierung der Versammlung bzw. des Europäischen Parlaments, die zwar nie lineare Züge aufwies - so kam es etwa erst im März 1960, als der Christdemokrat Hans Furler gegen den Liberalen Gaetano Martino die Wahl gewann, wieder zu einer

62. M. FORSYTH, op.cit., S.19. 
Kampfabstimmung über die Präsidentschaft des Europäischen Parlaments - die aber in wellenartigen Bewegungen bis heute anhält. Anders als in den Prognosen der sozialwissenschaftlichen Studien der 1960er Jahre angenommen, entwickelte sich ,im ideologischen Bereich echtes parlamentarisches Verhalten" ${ }^{\prime 63}$ aber nur sukzessive. Vielmehr scheinen die gemeinsamen europäischen Überzeugungen einer Mehrheit der Parlamentarier sowie die soziale und weltanschauliche Heterogenität auch innerhalb der Fraktionen einer starken Ideologisierung, wie sie häufig in nationalen Parlamenten anzutreffen ist, entgegengewirkt zu haben. Aufgabe künftiger integrationsbezogener Parteien- und Parlamentarismusforschung in historischer Perspektive muss es vor diesem Hintergrund sein, neben der kompetenziellen Ausgestaltung auch die temporalen Binnenstrukturen in den Blick zu nehmen - mithin diejenigen Entwicklungsprozesse, die sich in Generationenmustern und biographischen Prägungen, aber auch in Erfahrungs- und Erwartungsstrukturen widerspiegeln.

63. H.-V. SCHIERWATER, op.cit., S.107. 